\title{
Qualidade física do solo das trilhas do parque estadual do Cerrado - PR
}

\section{Physical quality of the trails at the state park Cerrado - PR}

\author{
Jully Gabriela Retzlaf de Oliveira' ${ }^{1 *}$;oão Tavares Filho²; \\ Graziela Moraes de Cesare Barbosa ${ }^{3}$
}

\section{Resumo}

A atividade turística em unidades de conservação vem ganhando interesse e destaque. Contudo, o aumento do fluxo de pessoas nestas áreas pode colocar em risco a integridade ecológica desses ambientes preservados, pois, a intensificação da passagem de turistas nas trilhas pode acentuar a possibilidade de compactação do solo (degradação do solo). O presente trabalho tem por objetivo avaliar o efeito do trânsito de pessoas em alguns atributos físicos do solo das trilhas do Parque Estadual do Cerrado (PR), a fim orientar futuras ações de planejamento do uso e ocupação da área que concorram para preservação do ecossistema. Para a avaliação física do solo foram coletadas em pontos aleatórios, 5 amostras de solo fora da trilha (FTr) e na trilha (Tr) com estrutura preservada na profundidade de $0-20 \mathrm{~cm}$. Com essas amostras, determinou-se a densidade do solo, a porosidade total, a macro e microporosidade, a matéria orgânica e a estabilidade de agregados. As trilhas do parque estadual do Cerrado (PR) que recebem mais de 50 visitantes ao longo do mês, apresentaram degradação em relação à densidade e porosidade do solo, e não apresentaram degradação quanto ao teor de matéria orgânica e agregação do solo em relação á área de solo nativo.

Palavras-chave: Turismo, física do solo, degradação do solo

\begin{abstract}
The tourism in protected areas has been gaining attention and interest. However, the increased flow of people in these areas may endanger the ecological integrity of these environments preserved since the intensification of tourist crossing the tracks may enhance the possibility of soil compaction (soil degradation). This study aims to evaluate the effect of the movement of people in some soil physical properties of the State Park trails Cerrado (PR) to guide future actions in planning the use and occupation of the area that contribute to the preservation of the ecosystem. To evaluate the physical soil were collected at random points, 5 soil samples off track (FTr) and track (Tr) with preserved structure in the depth of $0-20 \mathrm{~cm}$. With these samples, we determined the bulk density, total porosity, macro and micro, organic matter and aggregate stability. The trails of the state park Cerrado (PR) receiving more than 50 visitors over the month, showed no degradation as the organic matter content and soil aggregation in relation to the area of native soil.
\end{abstract}

Key words: Tourism, soil physics, soil degradation

\footnotetext{
${ }^{1}$ Prof $^{\mathrm{a}}$. M.e. do Dept ${ }^{\mathrm{o}}$ de Geografia, Universidade Estadual do Norte do Paraná, UENP, Cornélio Procópio, PR. E-mail: jullygeo@ yahoo.com.br

2 Prof. Dr. do Dept ${ }^{\circ}$ de Agronomia, Universidade Estadual de Londrina, UEL, Londrina, PR. E-mail: tavares@uel.br

${ }^{3}$ Pesquisadora Dra . Do Instituto Agronômico do Paraná, IAPAR, Área de Física do Solo, Londrina, PR. E-mail: graziela_barbosa@ iapar.br

* Autor para correspondência
} 
A atividade turística em unidades de conservação vem ganhando preferência e destaque dentre outras formas de turismo, resultando no aumento da visitação em áreas naturais. Contudo, o aumento do fluxo de pessoas nestas áreas pode colocar em risco a integridade ecológica desses ambientes preservados, pois, a intensificação da passagem de turistas nas trilhas pode acentuar a possibilidade de compactação do solo, com reflexos negativos em termos ambientais, como por exemplo, a erosão do solo.

O Parque Estadual do Cerrado, que possui 420,40 ha (IAP, 2002), recebe mais de 50 visitantes ao longo do mês. Este parque está voltado à preservação da biodiversidade, dos recursos naturais existentes e do patrimônio paisagístico, com destaque para a vegetação de Cerrado, um dos últimos remanescentes desta vegetação no Estado do Paraná. Contudo, a trilha existente na área (que vai do centro de visitantes ao limite norte do Parque) mostra possíveis sinais de degradação (compactação), uma vez que em alguns trechos a água não infiltra e acumula-se na superfície por longos períodos, às vezes por mais de três dias.

A qualidade, do ponto de vista físico, está associada àquele solo que: a) permite a infiltração, retenção e disponibilização de água às plantas, córregos e subsuperfície; b) responde ao manejo e resiste à degradação; c) permite as trocas de calor e de gases com a atmosfera e raízes de plantas; e d) possibilita o crescimento das raízes (REICHERT; REINERT; BRAIDA, 2003). Neste contexto, o presente trabalho tem por objetivo avaliar o efeito do trânsito de pessoas em alguns atributos físicos de solo das trilhas do Parque Estadual do Cerrado (PR), a fim orientar futuras ações de planejamento do uso e ocupação da área que concorram para preservação do ecossistema.

O Parque, pertencente ao município de Jaguariaíva - PR, está situado entre as coordenadas geográficas de $24^{\circ} 08^{\prime}$ e $24^{\circ} 11^{\prime}$ de Latitude Sul e $49^{\circ} 44^{\prime}$ e $49^{\circ} 39^{\prime}$ de Longitude Oeste (IAP, 2002).
O clima predominante na região é o $\mathrm{Cfb}$, na classificação de Koeppen, o solo é um Latossolo Vermelho distrófico e, a vegetação dominante é a de Cerrado (IAP, 2002; MAACK, 2002). Para a avaliação física do solo amostras foram coletadas dentro e fora de uma trilha utilizada para visitação há mais de dez anos, com uma freqüência de visitação de mais de 50 visitantes por mês.

Para a coleta das amostras, percorreu-se a trilha buscando os locais onde, aparentemente, existia maior problema de degradação (infiltração mais lenta de água no solo). Delimitaram-se duas extensões de $500 \mathrm{~m}$ cada e, em cada uma delas, bem como paralelamente a elas (na vegetação de cerrado), foram coletadas em pontos aleatórios, 5 amostras de solo fora da trilha (FTr) e na trilha (Tr) com estrutura preservada na profundidade de 0-20 cm. Com essas amostras, determinou-se a densidade do solo, a porosidade total, a macro e microporosidade e a matéria orgânica segundo Claessen et al. (1997), e a estabilidade de agregados, segundo Castro Filho, Muzilli e Podanosch (1998). A densidade do solo obtida por meio do método do anel volumétrico e a macro e microporosidade obtida por meio do método mesa de tensão, onde as amostras foram submetidas a uma tensão de $60 \mathrm{~cm}$ de coluna de água.

Para a estabilidade de agregados foram feitas 3 repetições de laboratório para cada amostra colatada a campo. Utilizaram-se as peneiras de malhas $8,0-$ $4,0-2,0-1,0-0,5$ e $0,25 \mathrm{~mm}$ de diâmetro. $\mathrm{O}$ solo retido em cada peneira foi quantificado, inclusive os agregados menores que $0,25 \mathrm{~mm}$, subtraindo-se o somatório do peso das outras classes de agregados do peso total da amostra seca. Obteve-se assim 6 classes de agregados, cujos diâmetros médios foram, respectivamente, 13,5 - 6,0 - 3,0 - 1,250,375 e $0,125 \mathrm{~mm}$. As médias dos valores obtidos foram usadas para o cálculo do diâmetro médio ponderado (DMP), diâmetro médio geométrico (DMG) e índice de estabilidade de agregados (IEA). Cada um destes índices apresenta um princípio diferente: o DMP aumenta seu valor quanto maior 
for a percentagem de agregados grandes retidos nas peneiras com malhas maiores; o DMG representa a estimativa do tamanho da classe de agregados de maior ocorrência; o IEA é uma medida de agregação total do solo, não considerando a distribuição por classes de agregados. Este índice será menor quanto maior a quantidade de agregados $<0,25 \mathrm{~mm}$.

Estes índices foram obtidos através das seguintes fórmulas:

$$
\mathrm{DMP}=\sum_{i=1}^{n}(x i . w i)
$$

onde: wi $=$ proporção de cada classe em relação ao total; $\mathrm{xi}=$ diâmetro médio das classes $(\mathrm{mm})$;

$$
\mathrm{DMG}=\mathrm{EXP} \frac{\sum_{l=1}^{N} w p \cdot \log x i}{\sum_{l=1}^{N} w p}
$$

onde: $\mathrm{wp}=$ peso dos agregados de cada classe (g);

$$
\mathrm{IEA}=\left(\frac{P A S-w p 25-\text { areia }}{P A S-\text { areia }}\right)
$$

onde: wp25 $=$ peso seco dos agregados da classe $<0.25 \mathrm{~mm}$; PAS = peso da amostra seca. Para o cálculo do IEA foi descontada a areia de acordo com Castro Filho, Muzilli e Podanosch (1998).

Utilizou-se $\mathrm{o}$ teste $\mathrm{t}$ para as amostras independentes com variâncias homogêneas, para comparar as médias das propriedades físicas entre vegetação nativa de Cerrado e Trilha.

Na Figura 1 estão apresentados os valores médios da matéria orgânica (MO), densidade do solo (Ds), volume total de poros (VTP), macroporosidade (Ma) e microporosidade (Mi) obtidos na área sob vegetação nativa de Cerrado fora da trilha (FTr) e na trilha (Tr), do Parque Estadual do Cerrado. Observase que o teor médio de matéria orgânica foi de 43,44 $\mathrm{g} \mathrm{dm}^{-3}$ para as amostras FTr e de $37,35 \mathrm{~g} \mathrm{dm}^{-3}$ para as amostras $\mathrm{Tr}$, sem ser observado diferença no nível de 5\% entre as mesmas; provavelmente devido ao grande intervalo de confiança e coeficiente de variação detectados nas amostras FTr.

Como se sabe, a matéria orgânica tem influência na agregação do solo; logo se pressupõem que quanto mais matéria orgânica, melhor a agregação, estabilidade dos agregados e distribuição do tamanho dos poros (BRAIDA et al., 2011). Segundo Wohlenberg et al. (2004), para manter a estrutura adequada ao desenvolvimento das plantas, circulação de ar e infiltração da água no solo é necessário o acréscimo contínuo de material orgânico. É importante lembrar também que a baixa densidade dos resíduos orgânicos, associada com sua susceptibilidade à deformação e elasticidade, torna-os potencialmente capazes de atenuar as cargas aplicadas ao solo (atenuar a compactação nas trilhas) (BRAIDA et al., 2011).

A densidade média do solo foi de $1,09 \mathrm{Mg} \mathrm{m}^{-3}$ para as amostras coletadas fora da trilha (FTr) e de $1,19 \mathrm{Mg} \mathrm{m}^{-3}$ para as amostras colatadas na trilha ( $\mathrm{Tr}$ ), existindo diferença no nível de 5\% entre as mesmas (Figura 1). Esse resultado indica ocorrência de compactação nas amostras coletadas da trilha, com prováveis prejuízos à infiltração da água no solo, pois, segundo Alves, Suzuki e Suzuki (2007), quando ocorre a degradação do solo em relação à densidade, o efeito imediato do seu aumento é a redução da macroporosidade, responsável pela infiltração e drenagem da água no solo. 
Figura 1. Valores médios, intervalos de confiança (95\%) e coeficiente de variação (CV) da matéria orgânica (MO), densidade do solo (Ds), volume total de poros (VTP), macroporosidade (Ma) e microporosidade (Mi) obtidos na área sob vegetação nativa de Cerrado fora da trilha (FTr) e na trilha (Tr), do Parque Estadual do Cerrado. *Médias seguidas de mesma letra minúscula na coluna, não diferem entre si, no nível de 5\%, pelo teste $t$.
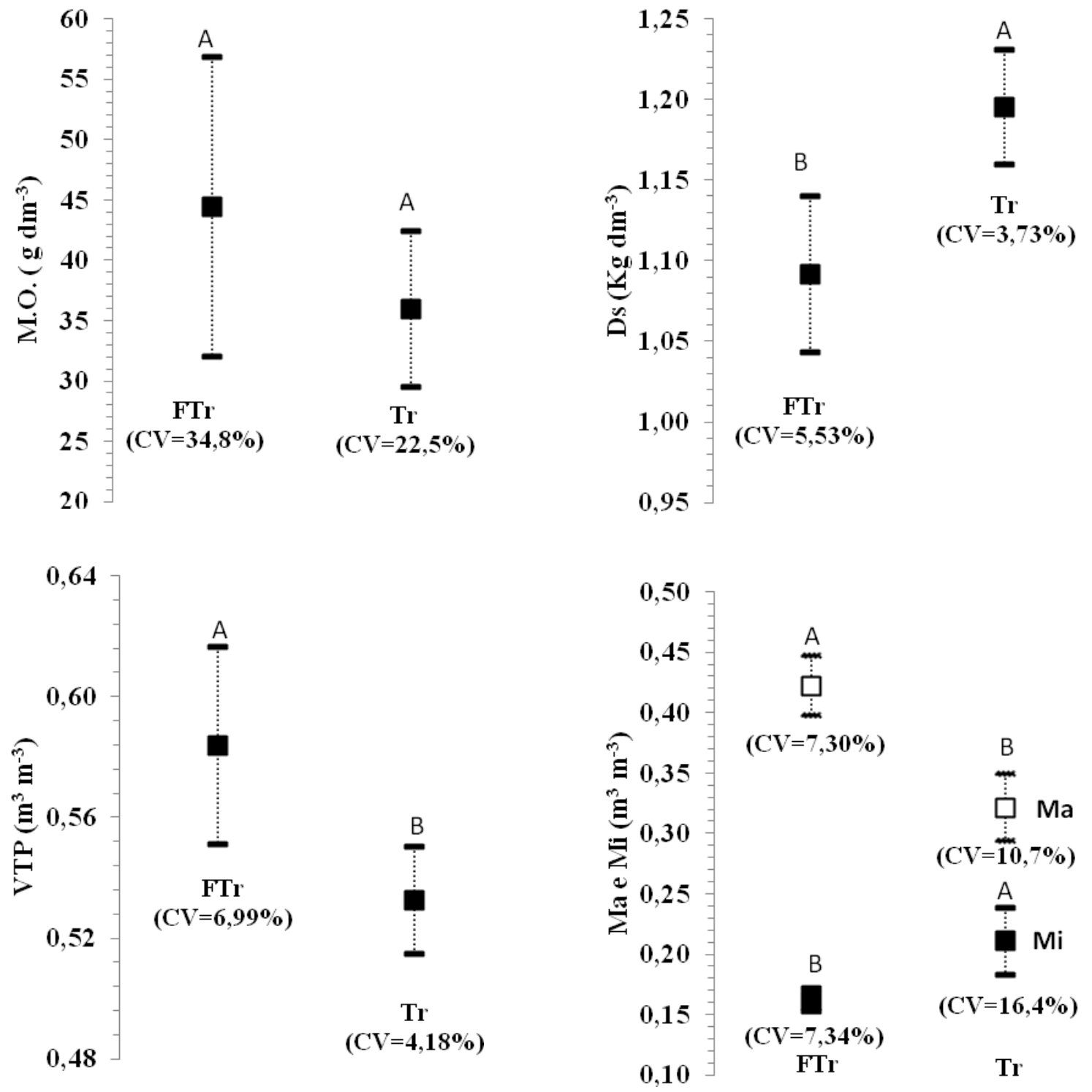

Fonte: Elaboração dos autores.

Os resultados obtidos estão em acordo com os dados apresentados por Vashchenko (2006); Maganhotto et al. (2010) e Saraiva (2011) os quais também encontraram valores de densidade do solo maiores nas trilhas em relação à área testemunha, para os picos Camapuã e Tucum, em trilha localizada na região de Paranaguá e em trilhas usadas pelo ecoturismo em Ubatuba, respectivamente. Por outro lado, Feola e Passos (2010) pesquisando processos erosivos na trilha noroeste do Parque Estadual do Pico do Marumbi, obtiveram valores de densidade do solo menor para trilha $\left(1,96 \mathrm{Mg} \mathrm{m}^{-3}\right)$ em relação à área testemunha $\left(2,02 \mathrm{Mg} \mathrm{m}^{-3}\right)$ na profundidade de $0-5 \mathrm{~cm}$, fato que se inverteu na profundidade de 
$5-10 \mathrm{~cm}$, sendo respectivamente $2,03 \mathrm{Mg} \mathrm{m}^{-3}$ e 1,98 $\mathrm{Mg} \mathrm{m}^{-3}$, sendo os primeiros resultados explicados pela diferença de densidade de partícula entre floresta e trilha.

Em relação as resultados da porosidade do solo, observa-se (Figura 1) que as amostras coletadas fora da trilha (FTr) apresentaram valores $(0,58$ (para VTP); 0,42 (para Ma) e 0,16 (para Mi) $\mathrm{m}^{3}$ $\mathrm{m}^{-3}$ ) com diferença no nível de 5\% em relação aos valores obtidos (0,53 (para VTP); 0,32 (para Ma) e 0,21 (para Mi) $\mathrm{m}^{3} \mathrm{~m}^{-3}$ ) para amostras colatadas na trilha (Tr), acarretando alterações do espaço poroso do solo que comprometem a infiltração da água no solo.

Saraiva (2011) constatou a diminuição da porosidade total do centro da trilha $0,44 \mathrm{~m}^{3} \mathrm{~m}^{-3} \mathrm{em}$ comparação à mata $0,64 \mathrm{~m}^{3} \mathrm{~m}^{-3}$, porém a autora afirma que estes valores estão de acordo com o intervalo adequado de variação para os solos de textura média. Também Maganhotto et al. (2010) verificaram diminuição da porosidade total na trilha em relação à área testemunho, com diminuição da macroporosidade e aumento da microporosidade.

Sabe-se que o aumento da densidade do solo (Ds $\operatorname{Tr}>$ Ds FTr - Figura 1) implica em redução da macroporosidade. Segundo Assouline, Tavares Filho e Tessier 1997; Tavares Filho e Tessier 2010, uma diferença de macroporosidade igual a $0,01 \mathrm{~m}^{3}$ $\mathrm{m}^{-3}$, resulta em $10 \mathrm{~m}^{3} \mathrm{Mg}^{-1}$ no volume de poros no solo. Portanto, os resultados apresentados mostram que o solo da trilha já perdeu cerca de $100 \mathrm{~m}^{3} \mathrm{Mg}^{-1}$ de seu volume de poros (a diferença entre os valores de macroporosidade é de $0,10 \mathrm{~m}^{3} \mathrm{~m}^{-3}$ ) devido ao transito de pessoas.
Considerando que os agregados presentes no solo são fatores de grande importância para a conservação do mesmo por conferirem maior resistência ao processo erosivo, proteção à matéria orgânica e, consequentemente, à população microbiana (FERREIRA; TAVARES FILHO; FERREIRA, 2010), na figura 2 estão apresentados os valores médios do diâmetro médio geométrico (DMG), do diâmetro médio ponderado (DMP) e índice de estabilidade de agregados (IEA) obtidos na área sob vegetação nativa de Cerrado fora da trilha (FTr) e na trilha (Tr), do Parque Estadual do Cerrado.

Observa-se (Figura 2) que os valores médios de DMG, DMP e IEA foram de 2,87, 1,36 mm e $89,30 \%$ respectivamente para as amostras $\mathrm{FTr}$ de $2,17,1,25 \mathrm{~mm}$ e $86,05 \%$ para as amostras $\mathrm{Tr}$, sem ser observado diferença no nível de 5\% entre as mesmas para os três índices analisados; provavelmente devido ao grande intervalo de confiança e coeficiente de variação detectados nas amostras avaliadas. Além disso, sabem-se que existe uma correlação entre matéria orgânica e agregação do solo, logo os resultados obtidos para a agregação do solo (Figura 2) podem ser um reflexo dos resultados apresentados na Figura 1, onde não foi observada diferença significativa entre as amostras coletadas fora e na trilha.

De acordo com os resultados obtidos, conclui-se que as trilhas do parque estadual do Cerrado (PR) que recebem mais de 50 visitantes ao longo do mês, apresentaram degradação em relação à densidade e porosidade do solo, e não apresentaram degradação quanto ao teor de matéria orgânica e agregação do solo em relação á área de solo nativo. 
Figura 2. Valores médios, intervalos de confiança (95\%) e coeficiente de variação (CV) do diâmetro médio geométrico (DMG), do diâmetro médio ponderado (DMP) e índice de estabilidade de agregados (IEA) obtidos na área sob vegetação nativa de Cerrado fora da trilha (FTr) e na trilha (Tr), do Parque Estadual do Cerrado. *Médias seguidas de mesma letra minúscula na coluna, não diferem entre si, no nível de 5\%, pelo teste $t$.
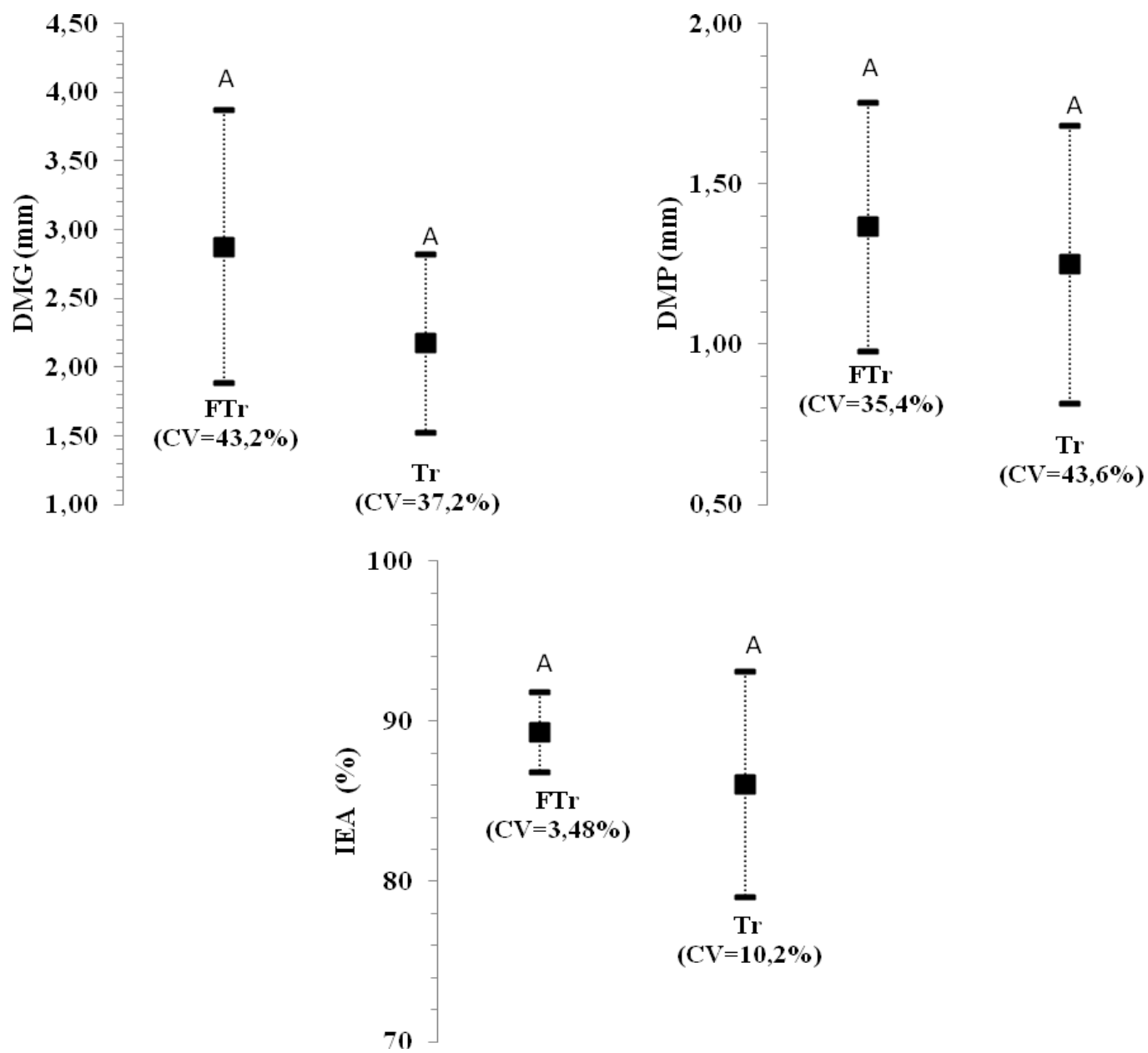

$(\mathrm{CV}=10,2 \%)$

Fonte: Elaboração dos autores.

\section{Agradecimentos}

Agradecemos ao Instituto Ambiental do Paraná (IAP), que permitiu a realização da pesquisa no parque, ao Instituto Agronômico do Paraná (IAPAR) que cedeu o laboratório de física do solo para serem feitas as análises deste trabalho.

\section{Referências}

ALVES, M. C.; SUZUKI, L. G. A. S.; SUZUKI, L. E. A. S. Densidade do solo e infiltração de água como indicadores da qualidade física de um latossolo vermelho distrófico em recuperação. Revista Brasileira de Ciência do Solo, Viçosa, n. 31, p. 617-625, 2007.

ASSOULINE, S.; TAVARES FILHO, J.; TESSIER, D. Effect of compaction on soil physical and hydraulic properties: experimental results and modeling. Soil Science Society of American Journal, Madison, v. 61, n. 2, p. 391-398, 1997. 
BRAIDA, J. A.; BAYER, C.; ALBUQUERQUE, J. A.; REICHERT, J. M. Matéria orgânica e seu efeito na física do solo. In: FILHO, O. K.; MAFRA, A. L.; GATIBONI, L. C. (Org.). Tópicos em ciência do solo. Viçosa: Sociedade Brasileira de Ciência do Solo, 2011. v. 7, p. 222-227.

CASTRO FILHO, C.; MUZILLI, O.; PODANOSCH, I. Estabilidade dos agregados e sua relação com o teor de carbono orgânico num Latossolo Roxo distrófico, em função de sistemas de plantio, rotações de cultura e métodos de preparo das amostras. Revista Brasileira de Ciência do Solo, Viçosa, v. 22, p. 527-538, 1998.

CLAESSEN, M. E. C.; BARRETO, W. O.; PAULA, J. L.; DUARTE, M. N. Manual de métodos de análise de solo. 2. ed. Rio de Janeiro: EMBRAPA, 1997. 212 p.

FEOLA, E.; PASSOS, E. Características Hidrológicas de um segmento de floresta ombrófila densa submontana: subsídio aos processos erosivos da trilha noroeste no Parque Estadual do Pico do Marumbi, Morretes-PR. Revista Brasileira de Geografia Física, Recife, v. 3, n. 2, p. 123-131, 2010.

FERREIRA, R. R. M.; TAVARES FILHO, J.; FERREIRA, V. M. Efeitos de sistemas de manejo de pastagens nas propriedades físicas do solo. Semina: Ciências Agrárias, Londrina, v. 31, n. 4, p. 913-932, out./dez. 2010.

MAACK, R. Geografia física do Estado do Paraná. 3. ed. Curitiba: Imprensa Oficial, 2002.

MAGANHOTTO, R. F.; SANTOS, L. J. C.; SOUZA, L. C. P.; MIARA, M. A. Variação dos atributos físicos do solo devido ao trânsito de pessoas em trilha localizada na Região de Paranaguá - Pr. Revista Geografar, Curitiba, v. 5, n. 2, p. 94-114, jul./dez. 2010.
INSTITUTO AMBIENTAL DO PARANÁ - IAP. Plano de manejo do parque estadual do cerrado. Curitiba: Secretaria do Estado do meio Ambiente e Recursos Hídricos, 2002.

REICHERT, J. M.; REINERT, D. J.; BRAIDA, J. A. Qualidade dos solos e sustentabilidade de sistemas agrícolas. Revista Ciência \& Ambiente, Santa Maria, v. 27, p. 29-48, 2003.

SARAIVA, A. C. R. Impactos aos atributos físicos do solo em trilhas ocasionados pelo ecoturismo em Ubatuba-SP. Revista Univap, São José dos Campos, v. 17, n. 29, p. 32-40, ago. 2011

TAVARES FILHO, J.; TESSIER, D. Effects of different management systems on porosity of Oxisols in Paraná, Brazil. Revista Brasileira de Ciência do Solo, Viçosa, v. 34, n. 3, p. 899-906, 2010.

VASHCHENKO, Y. Caracterização da trilha e o impacto do montanhismo nos Picos Camapuã e Tucum: Campina Grande do Sul, PR. 2006. Dissertação (Mestrado em Ciências Agrárias) - Universidade Federal do Paraná, Curitiba.

WOHLENBERG, E. V.; REICHERT, J. M.; REINERT, D. J.; BLUME, E. Dinâmica da agregação de um solo franco-arenoso em cinco sistemas de culturas em rotação e em sucessão. Revista Brasileira de Ciência do Solo, Viçosa, v. 28, n. 5, p. 891-900, 2004. 
